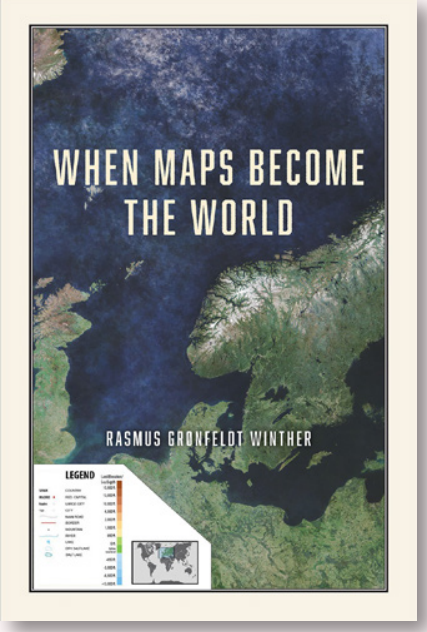

\title{
WHEN MAPS BECOME THE WORLD
}

\author{
By Rasmus Grønfeldt Winther
}

The University of Chicago Press, 2020

318 pages, with maps, satellite imagery, photography, and 10 color plates

Softcover: \$37.50, ISBN 978-0-226-67472-8

Review by: Daniel G. Cole, Smithsonian Institution

ReAding This воок, I could not help but be reminded of All Possible Worlds by Preston James (1972), the book we were required to read for a class in the history and philosophy of geography that I took back in my graduate school days. Of course, James was a geographer, writing about the philosophic history of his speciality; Winther, on the other hand, professes philosophy at University of California, Santa Cruz, and it was his love of maps that prompted him to write philosophically about cartography. Fully aware that he is exploring waters that may at times be deeper than some of his readers care to plumb, Winther provides helpful "swimmer's depth" ranking icons, "philosophical deep diving or philosophical snorkeling ... or easy reading" (xii) for each section of his text. These humble guideposts allow readers with differing interests and nuance tolerances to judge which passages they might safely plunge into or wisely avoid. There are also extensive footnotes throughout the book, supplying much needed information to aid readers who have done little wading in philosophy.

The first chapter, "Introduction: Why Maps?" presents the fundamental importance of maps for "finding our way in the world" (1), and continues with a short discussion about maps in both real and fictional worlds. Winther then lines up one of the central theses of his book: "... maps are abstractions discarding detail, focusing only on essential features of the territory. What is essential depends on one's purpose. ... In order to realize that a map is not the territory, we can, for instance, consider multiple points of view on-multiple maps of - the same territory" (3). Winther further posits that "this book is about the power and limitations of maps and mapping, including those ambitious and interconnected maps that we call scientific theories" (3).

From this beginning, the author addresses the history and philosophy of map thinking up until the present day (including GIS), and then compares and contrasts what he sees as the implicit worldviews of three maps: Waldseemüller's 1507 world map; Guaman Poma's Andean map included in his late sixteenth-century critique of Spanish colonial rule-which Winther identifies as a counter-map; and Tom Van Sant's 1990 geosphere map. The author concludes this introduction with a short discussion of Google Maps and Google Earth, and his hope that future cartographers will "reconstitute" the world in a new map, though Winther's meaning is unclear.

Chapter 2, "Theory is to World as Map is to Territory," presents a typology of map analogies-basic, general, extreme-scale, state-space, literal, causal, model, and paradigm map. Most of rest of the chapter involves some of the ways the philosophies of other disciplines employ map analogies or otherwise tie into maps. Winther shares the concern that Robinson and Petchenik raised in their classic 1976 book, The Nature of Maps "about what a map is or is good for," because, he writes, "maps do not just make the world. They help make other worlds, and the worlds of others" (52). 
Chapter 3, "From Abstraction to Ontologizing," looks at "cartographic practices to explore how representations are produced," with the goal of examining "the internal workings of abstraction and ontologizing practices" (59). Winther sees the abstraction of the world as taking place "via measurement and conceptualization to a representation" on paper or on a screen, and takes ontologizing to mean "deploying representation to do work in the world" (60). In this regard, the author takes particular note of the work of Robinson and Petchenik (1976) and that of Alan MacEachren (2004) in advancing cartographic communication. He then goes on to discuss the various "stages" of abstraction-calibration of units and coordinates, data collection and management, and generalization-and in this last mentioned stage, he covers the five "protocols" of generalization-selection of scale and projection, simplification, classification, symbolization, and exaggeration. The last pages of this chapter cover a broad range of topics. These include drawing parallels between his five cartographic generalization protocols to analogous practices in the social sciences, and a discussion of two broad approaches to abstraction-perspectivizing and partitioning-before finishing with "ontologizing representation testing," by which he means: changing the world, understanding the world, and classroom communication.

Chapter 4, "Long Live Contextual Objectivity!" deals with the what Winther calls the "pernicious reification" of maps, through which they cause readers to imagine the Earth as being the same shape as the map. He offers, as an example, a Ming dynasty map of China, that shows the Middle Kingdom placed in the center of the map, which may lead readers to believe that it truly is the center of the world. This is followed by a discussion on "contextual objectivity" - which he defines as "the quality resulting from good and proper application of a representation" (95) — with examples of county maps to show boundaries and a geologic map to illustrate prehistoric connections. Winther then provides some worthwhile quotations from other writers such as Ronald Giere (1999, 82): "The fit between a model and the world may be thought of like the fit between a map and the region it represents." The last seventeen pages of this chapter deal with the history of the Mercator projection and criticisms of its sometimes inappropriate use. He rightfully bemoans the use of Web Mercator as the default online map projection used by Google, Bing, and ArcGIS, but he does point out that "finding a projection that satisfies critiques of all perspectives ... is impossible. No single map can fully represent the world" (103).
Chapter 5, "Projecting Maps into Our Worlds," deals with the concepts of isomorphism (equal form) and similarity. Winther points out that "scale makes map space and world space isomorphic" (122), and that this state "is achieved with the equation characterizing the map projection" (125). Under the heading of similarity, the author discusses three types of symbols: abstract / geometric, mimetic, and pictographic. He later notes that both the metric and symbolic layers he sees maps as possessing "are concerned with assumptions about how to interpret and present the world in the map itself ..." (127). Further to this, he declares that "mapmakers perspectivize reality. That is, they impose a holistic, consistent perspective to make sense of a complex and finicky world" (128). Winther goes on to describe the experience of a map reader's abstracted viewing versus the direct cognitive cartography developed by a pedestrian traversing the same ground. This leads to a warning that "a map's cartopower can lead to its pernicious reification" (129) - a danger for which he declares counter-mapping to be the savior, as it brings light to alternative maps and minority viewpoints. At the chapter's end is a discussion on modeling climate change.

Chapter 6, "Mapping Space," covers a variety of topics, from mapping the universe in $3 \mathrm{D}$, to geologic mapping. On the latter subject, it includes a history of the theory of continental drift, culminating in the ocean floor maps Marie Tharp created between the 1950s and 1970s. Winther also presents sections on state-space maps in physics and physical chemistry, and analogous maps in mathematics. He concludes this section with a note that mapping "is a transformative process of establishing robust relations between representations, or between a representation and an ontologized world" (174).

Chapter 7, "Mapping Ourselves," is something of a catchall. It starts with a discussion of cartographic reification via European explorations from 1492 onwards. From this point, he covers a variety of topics, including migration maps, brain maps, and statistical causal maps. He wisely points out that the flow arrows found in migration maps "treat all people in a particular migration as similar" (181). This is due, at least in part, to a number of limiting cartographic parameters, but that it can be overcome has been shown through recent challenges posed by counter-mapping practices. In his section on brain maps, one subsection deals with counter-maps of the brain, or "cognitive ontologies." Surprisingly, while Winther briefly discusses cognitive mapping elsewhere in When Maps Become the World, it is not directly addressed here-nor is there any 
citation of Gould and White's Mental Maps (1974), or of any other similar work on cognitive cartography. The last section on statistical causal maps covers topics such as linear model assumptions, correlation and causation, genetic and environmental diseases, path diagrams as statistical causal maps, and when causal maps become the world. Needless to say, in this last subsection, the author points out how we shouldn't be defined by statistics, and that counter-mapping is possible.

Chapter 8, "Mapping Genetics," starts with Gregor Mendel's ground-breaking study on peas. From this point, Winther notes that genetic maps use a partitioning frame to track events over space and time. He then describes several types of genetic maps, before finishing with a look forward to mapping genetics as a "paradigmatic integration platform." Because of the technical scientific nature of this chapter, the author includes a glossary of genetic terms to assist any non-genetics-oriented readers.

The ninth and final chapter, "Map Thinking, Science, and Philosophy," serves as a summary for the rest of the book. In it, Winther states that "maps provide an intuitive window into how humans think and act, and steer their hopes and fears" (243). In the first section, titled "Existence, World Making and Responsibility," he addresses the concepts of constructionism, empiricism, and realism, and notes that "undeniably, map thinking invites us to entertain a plurality of philosophical projections" (248). Later, he follows with the observation that "just as there is no single correct map or map projection, or even a single correct and universal map abstraction and ontologizing practice, so there is no single way of interpreting or of practicing scientific methodology" (251). Winther concludes with a discussion of how map thinking "gives additional clarity to standard philosophical accounts of ethical thinking" (252). From this, he proposes what maps are good for: they provide what-if scenarios for imagining new kinds of worlds.

While When Maps Become the World is an engaging and informative work, its execution nonetheless has a few factual errors that tend to disrupt the flow of Winther's presentation. Many of these issues crop up in Chapter 3.

In the "Abstraction" section of that chapter, he makes several mistakes that might confuse a neophyte. It seems likely, for example, that his subsection title "Geodesic Surveying" (63) should more properly have been "Geodetic Surveying" instead. Similarly, on page 67 he refers to the
Defense Mapping Agency as if that agency had not ceased to exist in 1996-despite showing, on page 24, that he clearly knows about its current successor, the National Geospatial-Intelligence Agency. Winther's text also shows his inadequate investigation of certain cartographic fundamentals. One wonders why he even brings up the topic of hillshading, for example, when his only note on it-note 48 on page 78 - is an outdated reference from 2002. More seriously, the only equal-area projection he discusses is the little-used and widely panned Gall-Peters. For example, an exercise in the Appendix features two blank continental world maps-one in Mercator and the other in GallPeters. While I understand the choice of Mercator, why did he choose to ignore all of the other available (and widely used) equal-area projections such as Mollweide, Eckert IV, Equal Earth, and so on? Juxtaposing the Mercator and Gall-Peters seems just a little too much like a rehashing of Arno Peters's straw-man argument.

Finally, at the end of Chapter 6, on page 139, there is a discussion of climate change, but the pertinent figure referenced (Figure 5.1) is on page 118 - twenty-one pages away, and at the start of this chapter!

Nonetheless, I recommend this book to graduate students taking a class on the history and philosophy of geography, along with anyone else interested in this field. While I presented a number of problems with the book, they are essentially quibbles about minor confusions that can easily be puzzled out with careful reading. One hopes they will be dealt with in the next edition.

\section{REFERENCES}

Giere, Ronald N. 1999. Science without Laws. Chicago: University of Chicago Press.

Gould, Peter, and Rodney White. 1974. Mental Maps. Harmondsworth, UK: Penguin Books.

James, Preston E. 1972. All Possible Worlds: A History of Geographical Ideas. New York: Odyssey Press.

MacEachren, Alan M. 2004. How Maps Work: Representation, Visualization, and Design. New York: Guilford Press.

Robinson, Arthur H., and Barbara B. Petchenik. 1976. The Nature of Maps: Essays toward Understanding Maps and Mapping. Chicago: University of Chicago Press. 